\title{
Constraints on Radon and SGD Temporal Variability in a Semiarid, Well-mixed Estuary
}

\author{
WILLIAM WOLFE ${ }^{1}$ AND DORINA MURGULET ${ }^{2}$
}

${ }^{1}$ Texas A\&M University - Corpus Christi

${ }^{2}$ Texas A\&M University-Corpus Christi

Presenting Author: wwolfe1@islander.tamucc.edu

Submarine groundwater discharge (SGD) is defined as any and all types of water flow from the seabed to the coastal ocean, which includes both terrestrial/groundwater and oceanic/recirculated components. Although diffuse, and difficult to measure, SGD represents a significant source of freshwater and solutes to near-shore marine environments. Because radioisotopes like ${ }^{222} \mathrm{Rn}\left(\mathrm{t}_{1 / 2}=3.82\right.$ days $)$ and its parent, ${ }^{226} \mathrm{Ra}\left(\mathrm{t}_{1 / 2}\right.$ 1600 years), are more concentrated in groundwater than surface waters, they are ideal tracers of SGD in marine environments. Here we measure surface water variability of tracer activity in response to atmospheric and hydrologic factors. This multiseasonal study measures ${ }^{222} \mathrm{Rn}$ nearly continuously at a nearshore platform every 30 minutes since Summer of 2019, while ${ }^{226} \mathrm{Ra}$ is measured 1-3 times per week at the same site. Hydroparameters at the study site (wind speed, wind direction, groundwater and tide level, temperature, salinity and precipitation) are recorded simultaneously and correlated to tracer measurements. Initial results reveal trends that would otherwise be obscured over shorter study periods. Wind direction and velocity appear to be largely responsible for the observed radon variability. Additionally, tide and groundwater levels appear to be correlated to radon levels, often compounding or obscuring the wind effect. Long-term observations are analyzed using multi-linear regression, generalized additive modeling, and random forest regression. Results provide numerical relationships between environmental factors and tracer behavior. The correlations are integrated into a novel radon mass-balance for SGD estimates that accounts for atmospheric controls on tracer activity, reducing uncertainties associated with a traditional mass-balance approach. Additionally, these observations are used to create a prediction model for radon in surface water to better understand seasonal trends. 